\title{
Homotopy perturbation and Variational iteration methods for nonlinear fractional integro-differential equations
}

\author{
M.H.Saleh ${ }^{1}$, S.M.Amer ${ }^{1}$, A.S.Nagdy ${ }^{1}$, and M.E.Alngar ${ }^{1}$ \\ I'Mathematics Department, Faculty of Science, Zagazig University, Zagazig, Egypt)
}

\begin{abstract}
In this paper, the homotopy perturbation method (HPM) and variational iteration method (VIM) are applied to approximate solutions for nonlinear fractional integro-differential equations with boundary conditions. A comparison between these methods takes place. Numerical examples are presented to illustrate the efficiency and accuracy of the proposed methods.
\end{abstract}

Keywords: Boundary value problems, Caputo fractional derivative, Fractional integro-differential equations, Homotopy perturbation method and Variational iteration method.

\section{Introduction}

In recent years, fractional differential equations have attracted much more attention of physicists and mathematicians which provides an efficient for the description of many practical dynamical arising in engineering and scientific disciplines such as, physics, biology, chemistry, economy, electrochemistry, electromagnetic, control theory and viscoelasticity, see [1-6]. Many mathematical formulations of physical phenomena lead to integro-differential equations such as, fluid dynamics, continuum and statistical mechanics, see [7 - 9]. Integro-differential equations are usually difficult to solve analytically, so it is required to obtain an efficient approximate solution. The homotopy perturbation method and variational iteration method which are proposed by He [10 - 13] are of the methods which have received much concern. These methods have been successfully applied by many authors, such as the works in $[12,14,15]$.

In this paper, we applied the HPM and VIM for approximating the solution of nonlinear fractional integrodifferential equations of the second kind:

$D^{\alpha} y(x)-\lambda \int_{0}^{x} k(x, t) F(y(t)) d t=f(x), 0<x<b, 1<\alpha \leq 2$,

where $F(y(t))=[y(t)]^{q}, q>1$, subject to the boundary conditions

$y(0)=\gamma$,

$y(b)=\beta_{0}$,

where $D^{\alpha}$ indicates the Caputo fractional derivative, and $F(y(t))$ is a nonlinear continuous function, $\gamma, \beta_{0}$ are real constants, $f(x)$ and $k(x, t)$ are given that can be approximated by Taylor polynomials. The existence and stability of solutions for fractional integro-differential equations $[16,17]$. Also, in this paper we use the inverse operator $I^{\alpha}$ of $D^{\alpha}$ then the boundary conditions are used. There are many methods for seeking approximate solutions such as variational iteration method, homotopy perturbation method, homotopy analysis method, the fractional differential transform method and Adomian decomposition method. The outline of this paper is as follows: In section 2, we present some definitions. Section 3, contains the application of the homotopy perturbation method. Section 4, contains the application of the variational iteration method. Finally, section 5, devoted to illustrate some numerical examples on mentioned methods.

\section{Some Definitions And Notations}

Definition 2.1. A real function $f(x), x>0$, is said to be in the space $C_{\alpha}, \alpha \in R$, if there exists a real number $p>\alpha$, such that $f(x)=x^{p} f_{1}(x)$, where $f_{1}(x) \in C[0, \infty)$.

Definition 2.2. A real function $f(x), x>0$, is said to be in the space $C_{\alpha}^{k}, k \in N$, if $f^{k} \in C_{\alpha}$.

Definition 2.3. $I^{\alpha}$ denotes the fractional integral operator of order $\alpha$ in the sense of Riemann-Liouville, defined by:

$I^{\alpha} f(x)= \begin{cases}\frac{1}{\Gamma(\alpha)} \int_{0}^{x} \frac{f(t)}{(x-t)^{1-\alpha}} d t, & \alpha>0, \\ f(x), & \alpha=0 .\end{cases}$

Definition 2.4. Let $f \in C_{-1}^{m}, m \in N$. Then the Caputo fractional derivative of $f(x)$, defined by: 
$D^{\alpha} f(x)=\left\{\begin{array}{lc}\frac{1}{\Gamma(m-\alpha)} \int_{0}^{x} \frac{f^{m}(t)}{(x-t)^{\alpha-m+1}} d t, & 0 \leq m-1<\alpha \leq m, \\ \frac{d^{m} f(x)}{d x^{m}} & \alpha=m \in N .\end{array}\right.$

Now, we introduce some basic properties of fractional operator are listed below [1]:

For $f \in C_{\mu}, \mu \geq-1, \gamma \geq-1, \alpha, \beta \geq 0$ :

(1) $I^{\alpha} I^{\beta} f(x)=I^{\alpha+\beta} f(x)=I^{\beta} I^{\alpha} f(x)$.

(2) $I^{\alpha} x^{\gamma}=\frac{\Gamma(\gamma+1)}{\Gamma(\alpha+\gamma+1)} x^{\alpha+\gamma}$.

(3) $D^{\alpha}\left[I^{\alpha} f(x)\right]=f(x)$.

(4) $I^{\alpha}\left[D^{\alpha} f(x)\right]=f(x)-\sum_{k=0}^{m-1} f^{k}(0) \frac{x^{k}}{k !}, 0 \leq m-1<\alpha \leq m \in N . \quad$

\section{Homotopy Perturbation Method}

To illustrate the basic concepts of HPM for fractional Integro-differential equations, consider the equation (1) with boundary value conditions (2), (3). According to HPM [11 - 13], we construct the following homotopy:

$$
(1-P) D^{\alpha} y(x)+P\left(D^{\alpha} y(x)-\lambda \int_{0}^{x} k(x, t)[y(t)]^{q} d t\right)-f(x)=0,
$$

or

$$
D^{\alpha} y(x)=f(x)+P\left(\lambda \int_{0}^{x} k(x, t)[y(t)]^{q} d t\right),
$$

where $P \in[0,1]$ is an embedding parameter. If $P=0$, then equation (8) becomes,

$D^{\alpha} y(x)=f(x)$,

and when $P=1$, then the equation (8) becomes the original equation(1). The solution of equation (1)can be written as a power series in $P$ as follows:

$y(x)=y_{0}(x)+P y_{1}(x)+P^{2} y_{2}(x)+P^{3} y_{3}(x)+\cdots$

Put $P=1$ in equation (10), so the approximate solution of equation (1) is:

$y(x)=y_{0}(x)+y_{1}(x)+y_{2}(x)+y_{3}(x)+\cdots$

Substituting (10) in (8), and equating the coefficients of like powers of P, we have the following set of fractional differential equations:

$P^{0}: D^{\alpha} y_{0}(x)=f(x)$

$P^{1}: D^{\alpha} y_{1}(x)=\lambda \int_{0}^{x} k(x, t)\left[y_{0}(t)\right]^{q} d t$,

$P^{2}: D^{\alpha} y_{2}(x)=\lambda \int_{0}^{x} k(x, t)\left[y_{1}(t)\right]^{q} d t$,

$P^{3}: D^{\alpha} y_{3}(x)=\lambda \int_{0}^{x} k(x, t)\left[y_{2}(t)\right]^{q} d t$

Applying the inverse operator $I^{\alpha}$ of $D^{\alpha}$ to both sides of (12) - (15), the iterates are determined by the following recursive way:

$y_{0}(x)=\gamma+A x+I^{\alpha}[f(x)]$,

$y_{1}(x)=I^{\alpha}\left[\int_{0}^{x} k(x, t)\left[y_{0}(t)\right]^{q} d t\right]$,

Where $A=y^{\prime}(0)$.

The HPM series solutions generally converge very rapidly. For later numerical computation, let the expression: $\Phi_{N}(x)=y_{0}(x)+y_{1}(x)+y_{2}(x)+\cdots+y_{N-1}(x)$.

Denote the N-term approximation to $y(x)$. Now imposing the boundary conditions (3) on (18) we have the following equation:

$\Phi_{N}(b)=y_{0}(b)+y_{1}(b)+y_{2}(b)+\cdots+y_{N-1}(b)=\beta_{0}$.

From equation (19), we can find the unknowns $A$. Substituting the constant value of $A$ in equation (18), we have the approximate solution of the problem. 


\section{Variational Iteration Method}

Consider the fractional integro-differential equation (1) with boundary conditions (2), (3).According to VIM [18 - 20], we construct the correction functional for (1) as:

$y_{k+1}(x)=y_{k}(x)+I^{\beta}\left[\mu\left(D^{\alpha} y_{k}(x)-f(x)-\lambda \int_{0}^{x} k(x, p) F\left(\tilde{y}_{k}(p)\right) d p\right)\right]$,

or

$y_{k+1}(x)=y_{k}(x)+\frac{1}{\Gamma(\beta)} \int_{0}^{x}(x-s)^{\beta-1} \mu(s)\left(D^{\alpha} y_{k}(s)-f(s)-\lambda \int_{0}^{s} k(s, p) F\left(\tilde{y}_{k}(p)\right) d p\right) d s$,

where $I^{\beta}$ is the Riemann--Liouville fractional integral operator of order $\beta=\alpha+1-m, m \in N, \mu$ is a general Lagrange multiplier and $\widetilde{y}$ denotes restricted variation i.e. $\delta \tilde{y}_{k}=0$.

We make some approximation for the identification of an approximate Lagrange multiplier, so the correctional functional (21) can be approximately expressed as:

$y_{k+1}(x)=y_{k}(x)+\int_{0}^{x} \mu(s)\left(D^{2} y_{k}(s)-f(s)-\lambda \int_{0}^{s} k(s, p) F\left(\tilde{y}_{k}(p)\right) d p\right) d s$,

Making the above correction functional stationary, we obtain the following stationary conditions:

$1-\left.\mu^{\prime}(s)\right|_{x=s}=0,\left.\mu(s)\right|_{x=s}=0$. This gives the following Lagrange multiplier

$\mu(s)=(s-x)$.

We obtain the following iteration formula by substitution of (23) into functional (21),

$y_{k+1}(x)=y_{k}(x)+\frac{1}{\Gamma(\alpha-1)} \int_{0}^{x}(x-s)^{\alpha-2}(s-x)\left(D^{\alpha} y_{k}(s)-f(s)-\lambda \int_{0}^{s} k(s, p) F\left(\tilde{y}_{k}(p)\right) d p\right) d s$.

That is,

$y_{k+1}(x)=y_{k}(x)-\frac{\alpha-1}{\Gamma(\alpha)} \int_{0}^{x}(x-s)^{\alpha-1}\left(D^{\alpha} y_{k}(s)-f(s)-\lambda \int_{0}^{s} k(s, p) F\left(\tilde{y}_{k}(p)\right) d p\right) d s$.

This yields the following iteration formula:

$y_{k+1}(x)=y_{k}(x)-(\alpha-1) I^{\alpha}\left[D^{\alpha} y_{k}(x)-f(x)-\lambda \int_{0}^{x} k(x, p) F\left(\tilde{y}_{k}(p)\right) d p\right]$,

The initial approximation $y_{0}$ can be chosen by the following way which satisfies initial conditions (2):

$y_{0}(x)=\gamma+A x$,

where $A=y^{\prime}(0)$ is to be determined by applying suitable boundary conditions (3). We can obtain the following first-order approximation by substitution of (27) into (26):

$y_{1}(x)=y_{0}(x)-(\alpha-1) I^{\alpha}\left[D^{\alpha} y_{0}(x)-f(x)-\lambda \int_{0}^{x} k(x, p) F\left(\tilde{y}_{0}(p)\right) d p\right]$.

Similarly, we can obtain the higher-order approximations. If $N$ th-order approximate is enough, then imposing boundary conditions (3) in $N$ th-order approximation yields the following equation:

$Y_{N}(b)=\beta_{0}$.

From equation (29), we can find the unknown $A$. Substituting the constant values of $A$ in (29),we have the approximate solution of the problem.

\section{Examples}

In this section, we have applied homotopy perturbation method and variational iteration method for nonlinear fractional Integro-differential equations with known exact solution at $\alpha=2$. All the results are calculated by using the symbolic computation software Maple.

5.1. Example 1. Consider the following nonlinear fractional integro-differential equation:

$D^{\alpha} y(x)=2-\frac{1}{2} x^{3}-\frac{1}{2} x^{5}-\frac{1}{6} x^{7}+\int_{0}^{x} x t y^{2}(t) d t, 0<x<1,1<\alpha \leq 2$,

subject to the boundary conditions

$y(0)=1$,

$y(1)=2$.

For $\alpha=2$, the exact solution of (30) is given by

$y(x)=1+x^{2}$.

According to HPM, we construct the following homotopy:

$D^{\alpha} y(x)=2-\frac{1}{2} x^{3}-\frac{1}{2} x^{5}-\frac{1}{6} x^{7}+P\left[\int_{0}^{x} x t y^{2}(t) d t\right]$. 
Substituting (10) in (34), we obtain the following series of equations with identical power of $P$ :

$P^{0}: D^{\alpha} y_{0}(x)=2-\frac{1}{2} x^{3}-\frac{1}{2} x^{5}-\frac{1}{6} x^{7}$,

$P^{1}: D^{\alpha} y_{1}(x)=\int_{0}^{x} x t\left[y_{0}(t)\right]^{2} d t$,

$P^{2}: D^{\alpha} y_{2}(x)=\int_{0}^{x} x t\left[2 y_{0}(t) y_{1}(t)\right] d t$,

$P^{3}: D^{\text {á }} y_{3}(x)=\int_{0}^{x} x t\left[2 y_{0}(t) y_{2}(t)+y_{1}^{2}(t)\right] d t$,

Applying the operator $I^{\alpha}$ to the above series of nonlinear equations and using the initial condition (31), we get

$$
\begin{aligned}
& y_{0}(x)=1+A x+I^{\alpha}\left[2-\frac{1}{2} x^{3}-\frac{1}{2} x^{5}-\frac{1}{6} x^{7}\right], \\
& y_{1}(x)=I^{\alpha}\left[\int_{0}^{x} x t\left[y_{0}(t)\right]^{2} d t\right], \\
& y_{2}(x)=I^{\alpha}\left[\int_{0}^{x} x t\left[2 y_{0}(t) y_{1}(t)\right] d t\right]
\end{aligned}
$$

Where the constant $A=y^{\prime}(0)$. By solving (39) we obtain $y_{0}(x), y_{1}(x), \ldots$

$$
\begin{aligned}
& y_{0}(x)=1+A x+\frac{2 x^{\alpha}}{\Gamma(\alpha+1)}-\frac{3 x^{\alpha+3}}{\Gamma(\alpha+4)}-\frac{60 x^{\alpha+5}}{\Gamma(\alpha+6)}-\frac{840 x^{\alpha+7}}{\Gamma(\alpha+8)}, \\
& y_{1}(x)=I^{\alpha}\left[\int_{0}^{x} x t\left(1+A t+\frac{2 t^{\alpha}}{\Gamma(\alpha+1)}-\frac{3 t^{\alpha+3}}{\Gamma(\alpha+4)}-\frac{60 t^{\alpha+5}}{\Gamma(\alpha+6)}-\frac{840 t^{\alpha+7}}{\Gamma(\alpha+8)}\right)^{2} d t\right]
\end{aligned}
$$

Now, we can form the 2-term approximation:

$$
\begin{aligned}
\Phi_{2}(x)=y_{0}(x)+ & y_{1}(x) \\
=1+A x & +\frac{2 x^{\alpha}}{\Gamma(\alpha+1)}-\frac{3 x^{\alpha+3}}{\Gamma(\alpha+4)}-\frac{60 x^{\alpha+5}}{\Gamma(\alpha+6)}-\frac{840 x^{\alpha+7}}{\Gamma(\alpha+8)} \\
& +I^{\alpha}\left[\int_{0}^{x} x t\left(1+A t+\frac{2 t^{\alpha}}{\Gamma(\alpha+1)}-\frac{3 t^{\alpha+3}}{\Gamma(\alpha+4)}-\frac{60 t^{\alpha+5}}{\Gamma(\alpha+6)}-\frac{840 t^{\alpha+7}}{\Gamma(\alpha+8)}\right)^{2} d t\right],
\end{aligned}
$$

where $A$ can be determined by using the boundary condition (32) in $\Phi_{2}(x)$ (see Table 1). Table 2 shows the solution for different values of $\alpha$ by using HPM.

We compute the absolute error functions of HPM $E_{1}(x)=\left|\left(1+x^{2}\right)-\Phi_{2,1.25}\right|, E_{2}(x)=\left|\left(1+x^{2}\right)-\Phi_{2,1.5}\right|$, $E_{3}(x)=\left|\left(1+x^{2}\right)-\Phi_{2,1.75}\right|$, where $\left(1+x^{2}\right)$ is the exact solution of (30) - (32) and $\Phi_{2,1.25}, \Phi_{2,1.5}$ and $\Phi_{2,1.75}$ are approximate solutions of (30) - (32) by using (41) at $\alpha=1.25, \alpha=1.5$ and $\alpha=1.75$ respectively.

According to VIM, the formula (26) for (30) can be expressed in the following form:

$y_{k+1}(x)=y_{k}(x)-(\alpha-1) I^{\alpha}\left[D^{\alpha} y_{k}(x)-\left(2-\frac{1}{2} x^{3}-\frac{1}{2} x^{5}-\frac{1}{6} x^{7}\right)-\int_{0}^{x} x t\left[y_{k}(t)\right]^{2} d t\right]$,

Then, suppose that an initial approximation has the following form which satisfies the initial condition (31).

$y_{0}(x)=1+A x$.

Where the constant $A=y^{\prime}(0)$. Now by iteration formula (42), the first approximation takes the following form:

$y_{1}(x)=y_{0}(x)-(\alpha-1) I^{\alpha}\left[D^{\alpha} y_{0}(x)-\left(2-\frac{1}{2} x^{3}-\frac{1}{2} x^{5}-\frac{1}{6} x^{7}\right)-\int_{0}^{x} x t y_{0}^{2}(t) d t\right]$,

That is,

$y_{1}(x)=1+A x-(\alpha-1)\left[-\frac{2 x^{\alpha}}{\Gamma(\alpha+1)}+\frac{60 x^{\alpha+5}}{\Gamma(\alpha+6)}+\frac{840 x^{\dot{a}+7}}{\Gamma(\alpha+8)}-\frac{16 A x^{\alpha+4}}{\Gamma(\alpha+5)}-\frac{30 A^{2} x^{\alpha+5}}{\Gamma(\alpha+6)}\right]$, 
where $A$ can be determined by using the boundary condition (32) in $y_{1}(x)$ (see Table 3). Table 4 shows the solution for different values of $\alpha$ by using VIM.

We compute the absolute error functions of VIM $E_{4}(x)=\left|\left(1+x^{2}\right)-\mathrm{y}_{1,1.25}\right|, E_{5}(x)=\mid\left(1+x^{2}\right)-$ $\mathrm{y} 1,1.5, E 6 x=1+x 2-\mathrm{y} 1,1.75$, where $\left(1+x^{2}\right)$ is the exact solution of $(30)-(32)$ and y 1,1.25,y 1,1.5 and y 1 , 1.75 are approximate solutions of (30) - (32) by using (45) at $\alpha=1.25, \alpha=1.5$ and $\alpha=1.75$ respectively.

In Fig.1 we compare between the absolute errors functions of HPM and VIM.

5.2. Example 2. Consider the following nonlinear fractional integro-differential equation:

$D^{\alpha} y(x)=1-\int_{0}^{x} e^{t} y^{2}(t) d t, 0<x<1,1<\alpha \leq 2$,

subject to the boundary conditions

$y(0)=1$,

$y(1)=\mathrm{e}^{-1}$.

For $\alpha=2$, the exact solution of (46) is given by

$y(x)=\mathrm{e}^{-\mathrm{x}}$.

According to HPM, we construct the following homotopy:

$D^{\alpha} y(x)=1-P\left[\int_{0}^{x} e^{t} y^{2}(t) d t\right]$.

Substituting (10) in (50), we obtain the following series of equations with identical power of $P$ :

$P^{0}: D^{\alpha} y_{0}(x)=1$,

$P^{1}: D^{\alpha} y_{1}(x)=-\int_{0}^{x} e^{t}\left[y_{0}(t)\right]^{2} d t$,

$P^{2}: D^{\alpha} y_{2}(x)=-\int_{0}^{x} e^{t}\left[2 y_{0}(t) y_{1}(t)\right] d t$,

Applying the operator $I^{\alpha}$ to the above series of nonlinear equations and using the initial condition (47), we get: $y_{0}(x)=1+A x+I^{\alpha}[1]$,

$y_{1}(x)=-I^{\alpha}\left[\int_{0}^{x} e^{t}\left[y_{0}(t)\right]^{2} d t\right]$,

$y_{2}(x)=-I^{\alpha}\left[\int_{0}^{x} e^{t}\left[2 y_{0}(t) y_{1}(t)\right] d t\right]$

Where the constant $A=y^{\prime}(0)$. In order to avoid the difficult fractional integration and to show the efficiency of the present method for solving fractional integro-differential equations, we can simplify the integrations by taking the truncated Taylor expansions for the exponential term in (54): $e^{ \pm x} \approx 1 \pm \frac{x}{1 !}+\frac{x^{2}}{2 !} \pm \frac{x^{3}}{3 !}$.

By solving (54) we obtain $y_{0}(x), y_{1}(x), \ldots$.

$$
\begin{aligned}
& y_{0}(x)=1+A x+\frac{x^{\alpha}}{\Gamma(\alpha+1)}, \\
& y_{1}(x)=-I^{\alpha}\left[\int_{0}^{x}\left(1+\frac{t}{1 !}+\frac{t^{2}}{2 !}+\frac{t^{3}}{3 !}\right)\left(1+A t+\frac{t^{\alpha}}{\Gamma(\alpha+1)}\right)^{2} d t\right]
\end{aligned}
$$

Now, we can form the 2-term approximation:

$$
\begin{aligned}
\Phi_{2}(x) & =y_{0}(x)+y_{1}(x) \\
& =1+A x+\frac{x^{\alpha}}{\Gamma(\alpha+1)}-I^{\alpha}\left[\int_{0}^{x}\left(1+\frac{t}{1 !}+\frac{t^{2}}{2 !}+\frac{t^{3}}{3 !}\right)\left(1+A t+\frac{t^{\alpha}}{\Gamma(\alpha+1)}\right)^{2} d t\right],
\end{aligned}
$$

where $A$ can be determined by using the boundary condition (48) in $\Phi_{2}(x)$ (see Table 5). Table 6 shows the solution for different values of $\alpha$ by using HPM. 
We compute the absolute error functions of $\operatorname{HPM}_{7}(x)=\left|e^{-x}-\Phi_{2,1.25}\right|, E_{8}(x)=\left|e^{-x}-\Phi_{2,1.5}\right|, E_{9}(x)=$ $\left|e^{-x}-\Phi_{2,1.75}\right|$, where $e^{-x}$ is the exact solution of (46) - (48) and $\Phi_{2,1.25}, \Phi_{2,1.5}$ and $\Phi_{2,1.75}$ are approximate solutions of (46) - (48) by using (56) at $\alpha=1.25, \alpha=1.5$ and $\alpha=1.75$ respectively.

According to VIM, the formula (26) for (46) can be expressed in the following form:

$y_{k+1}(x)=y_{k}(x)-(\alpha-1) I^{\alpha}\left[D^{\alpha} y_{k}(x)-1+\int_{0}^{x} e^{t}\left[y_{k}(t)\right]^{2} d t\right]$,

Then, In order to avoid the difficult fractional integration and to show the efficiency of the present method for solving fractional integro-differential equations, we can simplify the integrations by taking the truncated Taylor expansions for the exponential term in (57): $e^{ \pm x} \approx 1 \pm \frac{x}{1 !}+\frac{x^{2}}{2 !} \pm \frac{x^{3}}{3 !}$.

Then suppose that an initial approximation has the following form which satisfies the initial condition (47).

$y_{0}(x)=1+A x$.

Where the constant $A=y^{\prime}(0)$. Now by iteration formula (57), the first approximation takes the following form:

$y_{1}(x)=y_{0}(x)-(\alpha-1) I^{\alpha}\left[D^{\alpha} y_{0}(x)-1+\int_{0}^{x} e^{t} y_{0}^{2}(t) d t\right]$.

That is,

$y_{1}(x)=1+A x-(\alpha-1)\left(-\frac{x^{\alpha}}{\Gamma(\alpha+1)}+I^{\alpha}\left[\int_{0}^{x}\left(1+\frac{t}{1 !}+\frac{t^{2}}{2 !}+\frac{t^{3}}{3 !}\right)(1+A t)^{2} d t\right]\right)$,

where $A$ can be determined by using the boundary condition (48) in $y_{1}(x)$ (see Table 7). Table 8 shows the solution for different values of $\alpha$ by VIM.

We compute the absolute error functions of VIM $E_{10}(x)=\left|e^{-x}-y_{1,1.25}\right|, \quad E_{11}(x)=\left|e^{-x}-y_{1,1.5}\right|$, $E_{12}(x)=\left|e^{-x}-\mathrm{y}_{1,1.75}\right|$, where $e^{-x}$ is the exact solution of (46) - (48) and $\mathrm{y}_{1,1.25}, \mathrm{y}_{1,1.5}$ and $\mathrm{y}_{1,1.75}$ are approximate solutions of (46) - (48) by using (60) at $\alpha=1.25, \alpha=1.5$ and $\alpha=1.75$ respectively.

In Fig. 2 we compare the absolute error functions of HPM and VIM.

\section{Figures And Tables}

Table 1.Values of the constants $A$ for different values of $\alpha$ using (41)

\begin{tabular}{ccccc}
\hline & $\alpha=1.25$ & $\alpha=1.5$ & $\alpha=1.75$ & $\alpha=2.0$ \\
\hline$A$ & -0.7790792844 & -0.5062608770 & -0.2427751792 & 0.0001542701076 \\
\hline
\end{tabular}

Table 2. Approximate solution of (30) for different values of $\alpha$ by HPM

\begin{tabular}{lllll}
\hline & $\chi=1.25$ & $\chi=1.5$ & $\chi=1.75$ & \\
\hline 0.0 & 1.000000000 & 1.0000000000 & 1.0000000000 & 1.000000000 \\
0.1 & 1.021357714 & 1.9969505189 & 1.9978354326 & 1.010015427 \\
0.2 & 1.080281359 & 1.0333139430 & 1.0258234010 & 1.040030854 \\
0.3 & 1.158228085 & 1.0953324640 & 1.0783842370 & 1.090046283 \\
0.4 & 1.250054387 & 1.1780994110 & 1.1530567910 & 1.160061714 \\
0.5 & 1.353185056 & 1.2787946790 & 1.2482728900 & 1.250077099 \\
0.6 & 1.466156193 & 1.3955466720 & 1.3628997220 & 1.360092113 \\
0.7 & 1.588093953 & 1.5270201170 & 1.4960612490 & 1.490105239 \\
0.8 & 1.718372500 & 1.6721984380 & 1.6470448770 & 1.640110962 \\
0.9 & 1.856206182 & 1.8301962800 & 1.8152302920 & 1.810091996 \\
1.0 & 2.000000000 & 2.0000000000 & 2.0000000000 & 2.000000000 \\
\hline
\end{tabular}

Table 3. Values of the constants $A$ for different values of $\alpha$ using (45)

\begin{tabular}{lllll}
\hline & $\alpha=1.25$ & $\alpha=1.5$ & $\alpha=1.75$ & $\alpha=2$ \\
\hline$A$ & 0.5605477271 & 0.2595474847 & 0.08288118852 & 0.01390932892 \\
\hline
\end{tabular}

Table 4. Approximate solution of (30) for different values of $\alpha$ by VIM.

\begin{tabular}{lllll}
\hline & $x=1.25$ & $x=1.5$ & $\chi=1.75$ & $x=2.0$ \\
\hline 0.0 & 1.000000000 & 1.000000000 & 1.000000000 & 1.000000000 \\
0.1 & 1.080871242 & 1.049743088 & 1.024872840 & 1.011390932 \\
0.2 & 1.171135364 & 1.119193616 & 1.072360292 & 1.042781733 \\
0.3 & 1.266160765 & 1.201475277 & 1.138277066 & 1.094170375 \\
0.4 & 1.364663434 & 1.294130685 & 1.220767259 & 1.165544888 \\
0.5 & 1.465984751 & 1.395713749 & 1.318609850 & 1.256861976 \\
0.6 & 1.569703107 & 1.505172737 & 1.430824772 & 1.368003465 \\
0.7 & 1.675470697 & 1.621568600 & 1.556453131 & 1.498699171 \\
0.8 & 1.782890263 & 1.743859428 & 1.694355584 & 1.648401434 \\
0.9 & 1.891379861 & 1.870669387 & 1.842971274 & 1.816092395 \\
1.0 & 2.000000000 & 2.000000000 & 2.000000001 & 2.000000000 \\
\hline
\end{tabular}


Table 5. Values of the constants $A$ for different values of $\alpha$ using (56)

\begin{tabular}{lllll}
\hline & $\alpha=1.25$ & $\alpha=1.5$ & $\alpha=1.75$ & $\alpha=2$ \\
\hline$A$ & -1.101981487 & -1.120200237 & -1.068558157 & -0.9973440064 \\
\hline
\end{tabular}

Table 6. Approximate solution of (46) for different values of $\alpha$ by HPM

\begin{tabular}{lllll}
\hline & $\alpha=1.25$ & $\alpha=1.5$ & $\alpha=1.75$ & $\alpha=2.0$ \\
\hline 0.0 & 1.0000000000 & 1.0000000000 & 1.0000000000 & 1.0000000000 \\
0.1 & 0.9372573741 & 0.9108427458 & 0.9038098154 & 0.9051029925 \\
0.2 & 0.8873411108 & 0.8381220095 & 0.8209156604 & 0.8192614267 \\
0.3 & 0.8397024746 & 0.7736949487 & 0.7474146156 & 0.7416113133 \\
0.4 & 0.7908043115 & 0.7142364634 & 0.6812126113 & 0.6713662027 \\
0.5 & 0.7384177754 & 0.6576138965 & 0.6207998324 & 0.6078050707 \\
0.6 & 0.6808045346 & 0.6022234665 & 0.5649667521 & 0.5502593763 \\
0.7 & 0.6164291922 & 0.5467317813 & 0.5126771966 & 0.4980991660 \\
0.8 & 0.5438210778 & 0.4899419936 & 0.4629937424 & 0.4507179262 \\
1.0 & 0.4614921978 & 0.4307095057 & 0.4150246141 & 0.4075156696 \\
\hline
\end{tabular}

Table 7.Values of the constants $A$ for different values of $\alpha$ using (60).

\begin{tabular}{ccccc}
\hline & $\alpha=1.25$ & $\alpha=1.5$ & $\alpha=1.75$ & $\alpha=2.0$ \\
\hline$A$ & -0.6465788051 & -0.6528627275 & -0.6535942959 & -0.6513580333 \\
\hline
\end{tabular}

Table 8. Approximate solution of (46) for different values of $\alpha$ by VIM.

\begin{tabular}{lllll}
\hline & $\alpha=1.25$ & $\alpha=1.5$ & $\alpha=1.75$ & $\alpha=2.0$ \\
\hline 0.0 & 1.0000000000 & 1.0000000000 & 1.0000000000 & 1.0000000000 \\
0.1 & 0.9465147794 & 0.9454228590 & 0.9421062944 & 0.9393655216 \\
0.2 & 0.8943468483 & 0.8963931622 & 0.8916315440 & 0.8857506248 \\
0.3 & 0.8405291699 & 0.8476312139 & 0.8437932991 & 0.8362104424 \\
0.4 & 0.7840458613 & 0.7966000207 & 0.7953958735 & 0.7878462359 \\
0.5 & 0.7243188124 & 0.7415534717 & 0.7438320925 & 0.7378136463 \\
0.6 & 0.6609792090 & 0.6811641977 & 0.6868513763 & 0.6833309391 \\
0.7 & 0.5937859545 & 0.6143785802 & 0.6224623676 & 0.6216869603 \\
0.8 & 0.5225868410 & 0.5403440458 & 0.5488819619 & 0.5502484321 \\
0.9 & 0.4472961608 & 0.4583661449 & 0.4645043544 & 0.4664661365 \\
1.0 & 0.3678794410 & 0.3678794412 & 0.3678794411 & 0.3678794412 \\
\hline
\end{tabular}
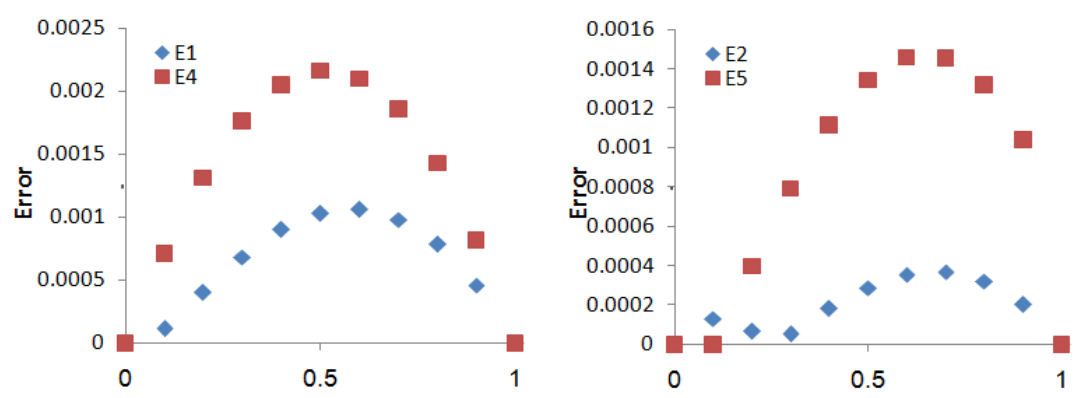

(1a) $E_{1}$ and $E_{4}$ for $\alpha=1.25$

(1b) $E_{2}$ and $E_{5}$ for $\alpha=1.5$

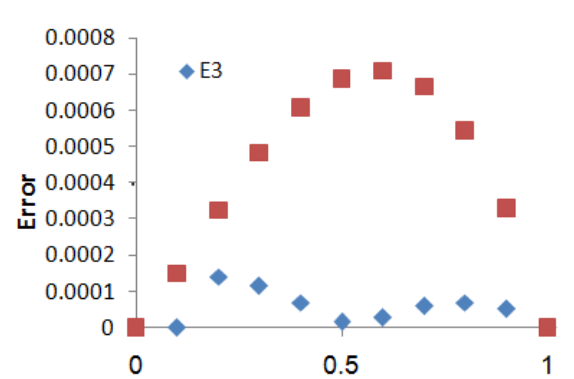

(1c) $E_{3}$ and $E_{6}$ for $\alpha=1.75$

Fig.1. Comparison of absolute error functions $E_{1}(x)-E_{6}(x)$ obtained by HPM and VIT for different $\alpha$. 


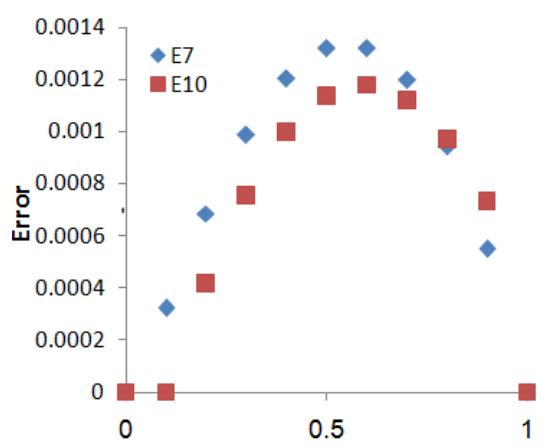

(2a) $E_{7}$ and $E_{10}$ for $\alpha=1.25$

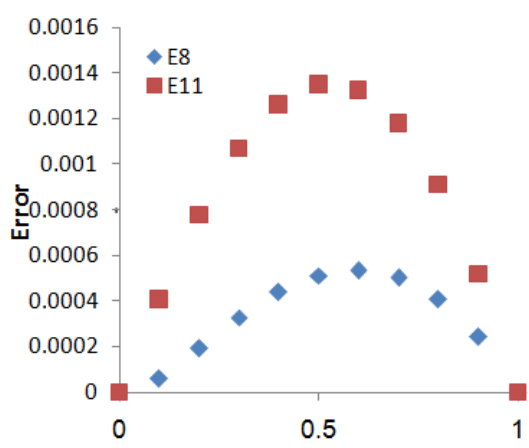

(2b) $E_{8}$ and $E_{11}$ for $\alpha=1.5$

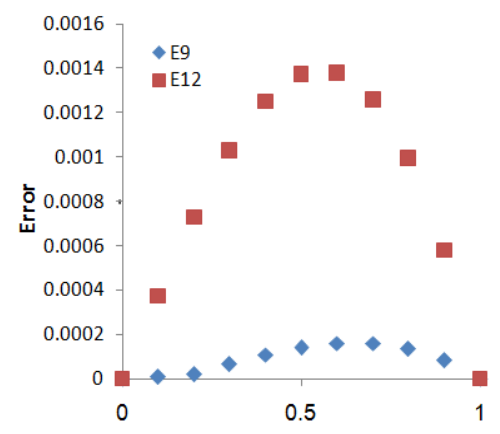

(1c) $E_{9}$ and $E_{12}$ for $\alpha=1.75$

Fig.2. Comparison of absolute error functions $E_{7}(x)-E_{12}(x)$ obtained by HPM and VIT for different 0

\section{Conclusion}

In this paper, this study showed that the numerical results of most nonlinear fractional integrodifferential equations (1) - (3). We usually derive very good approximations to the solutions. It can be concluded that the HPM and VIM are a powerful and efficient technique in finding very good solutions for this kind of equations. We find that HPM is better than VIM (see Fig.1 and Fig.2).Also it is shown that the accuracy can be improved by more $\mathrm{N}$-terms of approximated solutions and by taking more terms in the Taylor expansion of the exponential term. In our paper, we use the Maple Package to calculate the functions obtained from the HPM and VIM.

\section{References}

[1] A. A. Kilbas, H. M. Srivastava, and J. J. Trujillo, Theory and Applications of Fractional Differential Equations, vol. 204 of NorthHolland Mathematics Studies, Elsevier Science B.V., Amsterdam, The Netherlands, 2006

[2] V. Lakshmikantham, S. Leela, and J. V. Devi, Theory of Fractional Dynamic Systems, Cambridge Scientific, 2009.

[3] K. S. Miller and B. Ross, An Introduction to the Fractional Calculus and Fractional Differential Equations, A Wiley-Interscience Publication, John Wiley \& Sons, New York, NY, USA, 1993.

[4] I. Podlubny, Fractional Differential Equations, vol. 198 of Mathematics in Science and Engineering, Academic Press, San Diego, Calif, USA, 1999.

[5] K. Diethelm and A.D. Freed, "On the solution of nonlinear fractional order differential equations used in the modeling of viscoelasticity," in Scientific Computing in Chemical Engineering II-Computational Fluid Dynamics, Reaction Engineering and Molecular Properties, F. Keil, W. Mackens, H. Voss, and J. Werther, Eds., pp. 217--224, Springer, Heidelberg, Germany, 1999.

[6] R. Metzler, W. Schick, H.-G. Kilian, and T. F. Nonnenmacher, "Relaxation in filled polymers: a fractional calculus approach," Journal of Chemical Physics, vol. 103, no. 16, pp. 7180--7186, 1995.

[7] L. Gaul, P. Klein, and S. Kemple, "Damping description involving fractional operators," Mechanical Systems and Signal Processing, vol. 5 , no. 2 , pp. $81--88,1991$.

[8] R. Hilbert, Applications of Fractional Calculus in Physics, World Scientific, River Edge, NJ, USA, 2000.

[9] R. P. Agarwal, M. Benchohra, and S. Hamani, "A survey on existence results for boundary value problems of nonlinear fractional differential equations and inclusions," Acta ApplicandaeMathematicae, vol. 109, no. 3, pp. 973--1033, 2010.

[10] J.-H. He, "Variational iteration method---a kind of non-linear analytical technique: some examples," International Journal of NonLinear Mechanics, vol. 34, no. 4, pp. 699--708, 1999.

[11] J.-H. He, "Homotopy perturbation method: a new nonlinear analytical technique," Applied Mathematics and Computation, vol. 135, no. 1, pp. $73--79,2003$.

[12] J.-He, "Homotopy perturbation technique," Computer Methods in Applied Mechanics and Engineering, vol. 178, no. 3-4, pp. 257-$262,1999$.

[13] J.-H. He, "Homotopy perturbation method with an auxiliary term," Abstract and Applied Analysis, vol. 2012, Article ID 857612, 7 pages, 2012. 
[14] S. Abbasbandy, "An approximation solution of a nonlinear equation with Riemann-Liouville's fractional derivatives by He's variational iteration method," Journal of Computational and Applied Mathematics, vol. 207, no. 1, pp. $53--58,2007$.

[15] O. Abdulaziz, I. Hashim, and S. Momani, "Application of homotopy-perturbation method to fractional IVPs," Journal of Computational and Applied Mathematics, vol. 216, no. 2, pp. 574--584, 2008.

[16] Bashir Ahmad, Juan J. Nieto, Existence Results for Nonlinear Boundary Value problems of Fractional Integro-differential Equations with Integral Boundary Conditions, Hindawi Publishing Corporation, Boundary Value problems. (2009) 1-11.

[17] Shaher Momani, Samir Hadid, LYAPUNOV Stability Solutions of Fractional Integro-differential Equations, Hindawi Publishing Corporation, IJMMS. 47 (2004) 2503-2507.

[18] H. Jafari, A. Kadem, D. Baleanu, and T. Yilmaz, "Solutions of the fractional Davey-Stewartson Equations with variational iteration method," Romanian Reports in Physics, vol. 64, no. 2, pp. 337--346, 2012.

[19] A. Kadem and A. Kilic, man, "The approximate solution of fractional Fredholm integrodifferential equations by variational iteration and homotopy perturbation methods," Abstract and Applied Analysis, vol. 2012, Article ID 486193, 10 pages, 2012.

[20] Y. Nawaz, "Variational iteration method and homotopy perturbation method for fourth-order fractional integro-differential equations," Computers \& Mathematics with Applications, vol. 61, no. 8, pp. 2330--2341, 2011. 